\title{
Analysis of Generalized Exponential Distribution Under Adaptive Type-II Progressive Hybrid Censored Competing Risks Data
}

\author{
S. K. Ashour ${ }^{1}$, M. M. A. Nassar $*^{2}$ \\ ${ }^{l}$ Department of Mathematical Statistics, Institute of Statistical Studies \& research. Cairo University, Egypt \\ ${ }^{2}$ Department of Statistics, Faculty of Commerce, Zagazig University, Egypt \\ *Corresponding author E-mail: mezo10011@gmail.com
}

Copyright $\odot 2014$ S.K. Ashour, M.M.A.Nassar. This is an open access article distributed under the Creative Commons Attribution License, which permits unrestricted use, distribution, and reproduction in any medium, provided the original work is properly cited.

\begin{abstract}
This paper presents estimates of the parameters based on adaptive type-II progressive hybrid censoring scheme (AT-II PHCS) in the presence of the competing risks model. We consider the competing risks have generalized exponential distributions (GED). The maximum likelihood method is used to derive point and asymptotic confidence intervals for the unknown parameters. The relative risks due to each cause of failure are investigated. A real data set is used to illustrate the theoretical results and to test the hypothesis that the causes of failure follow the generalized exponential distributions against the exponential distribution (ED).
\end{abstract}

Keywords: Competing Risks; Adaptive Type-II Progressive Hybrid Censoring; Generalized Exponential Distribution; Maximum Likelihood Estimation.

\section{Introduction}

In the context of life testing experiments, hybrid censoring scheme was introduced at first by Epstein [3]. Since the introduction by Epstein [3], extensive works and different types of hybrid censoring scheme has been appeared. Recently, Kundu and Joarder [8] and Childs et al [2] both investigated the type-II progressive hybrid censoring scheme (T-II PHCS), where the life testing experiment with progressive censoring scheme $\left(R_{1}, R_{2}, \ldots, R_{m}\right)$ is terminated at time $T^{*}=\min \left\{X_{m: m: n}, T\right\}$, where $T \in(0, \infty)$ is the prefixed time and $X_{m: m: n}$ denotes the $m$-th failure time when $n$ items are place on a life test experiment. Briefly, If $X_{m: m: n}<T$ the experiment terminate at time $X_{m: m: n}$ and $m$ failures occurs; otherwise, the experiment stops at time $T$ and only $J$ failures occur before time $T$, where $X_{J: m: n}<T<X_{J+1: m: n}$ and $0 \leq J<m$.

The drawback of the T-II PHCS is that the effective number of failures is random and it can be a very small number (even equal to zero), so that usual statistical inference procedures will not be applicable or they will have low efficiency. For this reason, $\mathrm{Ng}$ et al [14] suggested an adaptive type-II progressive hybrid censoring scheme in which the effective number of failures $m$ is fixed in advance and the progressive censoring scheme $R_{1}, R_{2}, \ldots, R_{m}$ is provided, but the values of some of the $R_{i}$ may be change accordingly during the experiment. Suppose the experimenter provides a time $T$, which is an ideal total test, but the experimental time is allowed to run over time $T$. If the $m$-th progressively censored observed failures occurs before time $T$ (i.e. $X_{m: m: n}<T$ ), the experiment stops at this time $X_{m: m: n}$, and we will have a usual type-II progressive censoring scheme with the prefixed progressive censoring scheme $R_{1}, R_{2}, \ldots, R_{m}$. Otherwise, once the experimental time passes time $T$ but the number of observed failures has not reached $m$, then we adapt the number of items progressively removed from the experiment upon failure by setting $R_{J+1}, R_{J+2}, \ldots, R_{m-1}=0$ and $R_{m}=n-m-\sum_{i=1}^{J} R_{i}$, where $X_{J: m: n}<T<X_{J+1: m: n}$, and $X_{J: m: n}$ is the $J$-th failure time occur 
before time $T$ and $J+1<m$. Thus the effectively applied scheme is $R_{1}, \ldots, R_{J}, 0, \ldots, 0, n-m-\sum_{i=1}^{J} R_{i}$. This formulate on leads to terminate the experiment as soon as possible if the $(J+1)$-th failure time is greater than $T$, and the total test time will not be too far away from the time $T$. If $T=0$, the scheme will lead us to the case of conventional type-II censoring scheme, and if $T \rightarrow \infty$, we will have a usual progressive type-II censoring scheme.

We should mention here that many authors studied the statistical properties of some life time models under AT-II PHCS in the presence of one and two causes of failures. $\mathrm{Ng}$ et al [14] developed inferential methods for the case when the lifetime distribution is exponential. They observed that the MLE always exists in this case. Lin et al [5], considered the adaptive progressive censoring scheme when the lifetime distribution is Weibull, and discussed the corresponding inferential issues. They have also discussed confidence intervals for the model parameters through the use of the asymptotic distribution of the MLEs as well as by the bootstrap method. Hemmati and Khorram [11], studied the competing risk model based on exponential distributions under the adaptive type-II progressively censoring scheme. They obtained the maximum likelihood and the Bayes estimators of the exponential distribution parameter, and two sides Bayesian probability intervals of the parameter are also obtained. Hemmati and Khorram [10], obtained the maximum likelihood estimators of the parameters from a two-parameter log-normal distribution based on the adaptive Type-II progressive hybrid censoring scheme, they compared the results with corresponding estimators of the type-II progressive hybrid censoring scheme. Mahmoud et al [15], obtained the maximum likelihood estimators for the unknown parameters of Pareto distribution based on the adaptive type-II progressive censoring scheme, point estimation and confidence intervals based on maximum likelihood also proposed.

The main aim of this paper is analyzing the AT-II PHCS under the competing risk model when lifetimes have independent GED. We derive the maximum likelihood estimates (MLE) and we obtain the approximate two sided confidence intervals of these different parameters. We use the likelihood ratio test to test the ED against the GED. We consider a real data set and see how the different models work in the practical situation.

The rest of this paper is organized as follows: In section (2), we introduce the model and the notation used throughout this paper. In section (3), we discuss the maximum likelihood estimation; confidence intervals are presented in section (4). In section (5), Goodness of fit test for testing a competing risks model where causes follow a GED against ED. In section (6), a real data set is used to illustrate the theoretical results.

\section{Model description and notation}

In reliability analysis, the failure of items may be attributable to more than one cause at the same time. These "causes" are competing for the failure of the experimental unit. Consider a life time experiment with $n \in N$ identical units, where its lifetimes are described by independent and identically distributed (i.i.d) random variables $X_{1}, X_{2}, \ldots, X_{n}$. Without loss of generality; assume that there are only two causes of failure. We have $X_{i}=\min \left\{X_{1 i}, X_{2 i}\right\}$ for $i=1, \ldots, n$, where $X_{k i}, k=1,2$, denotes the latent failure time of the $i$-th unit under the $k$-th cause of failure. We assume that the latent failure times $X_{1 i}$ and $X_{2 i}$ are independent, and the pairs $\left(X_{1 i}, X_{2 i}\right)$ are i.i.d. Assume that the failure times follows the GED introduced by Gupta and Kundu [16] as generalization of the exponential distribution with the probability density function $f_{k}(x)$ as

$f_{k}(x)=\alpha_{k} \cdot \beta_{k} e^{-\alpha_{k} \cdot x} \cdot\left(1-e^{-\alpha_{k} \cdot x}\right)^{\beta_{k}-1}, x>0, \alpha_{k} \cdot \beta_{k}>0$

where $\alpha_{k}$ is the scale parameter and $\beta_{k}$ is the shape parameters. The cumulative distribution function $F_{k}(x)$ and failure hazard function $h_{k}(x)$ have the form

$F_{k}(x)=\left(1-e^{-\alpha_{k} \cdot x}\right)^{\beta_{k}}$

and

$h_{k}(x)=\alpha_{k} \cdot \beta_{k} \cdot e^{-\alpha_{k} \cdot x} \cdot\left(1-e^{-\alpha_{k} \cdot x}\right)^{\beta_{k}-1}\left[1-\left(1-e^{-\alpha_{k} \cdot x}\right)^{\beta_{k}}\right]^{-1}$

Under AT-II PHCS and in presence of competing risks data we have the following observation:

$$
\left(X_{1: m: n}, c_{1}, R_{1}\right), \ldots,\left(X_{J: m: n}, c_{J}, R_{J}\right),\left(X_{J+1: m: n}, c_{J+1}, 0\right), . .,\left(X_{m-1: m: n}, c_{m-1}, 0\right),\left(X_{m: m: n}, c_{m}, R_{m}\right)
$$

where $J=\max \left\{J: X_{J: m: n}<T\right\}, R_{m}=n-m-\sum_{i=1}^{J} R_{i}$ and $c_{i} \in(1,2)$. Here, $c_{i}=1,2$ means the unit $i$ has failed at time $X_{i: m: n}$ due to the first and the second cause of failures, respectively. Let

$I_{1}\left(c_{i}=1\right)=\left\{\begin{array}{ll}1, & c_{i}=1 \\ 0 & \text { else }\end{array}, I_{2}\left(c_{i}=2\right)= \begin{cases}1, & c_{i}=2 \\ 0 & \text { else }\end{cases}\right.$

thus the random variables $m_{1}=\sum_{i=1}^{m} I_{1}\left(c_{i}=1\right)$ and $m_{2}=\sum_{i=1}^{m} I_{2}\left(c_{i}=2\right)$ describe the number of failures due to the first and the second cause of failures, respectively and $m=m_{1}+m_{2}$. Now we can write the likelihood function of the observed data as follows 
$L=C \prod_{i=1}^{m}\left\{\left[f_{1}\left(x_{i}\right) \bar{F}_{2}\left(x_{i}\right)\right]^{I\left(c_{i}=1\right)}\left[f_{2}\left(x_{i}\right) \bar{F}_{1}\left(x_{i}\right)\right]^{I\left(c_{i}=2\right)}\right\} \prod_{i=1}^{J}\left\{\left[\bar{F}_{1}\left(x_{i}\right) \bar{F}_{2}\left(x_{i}\right)\right]^{R_{i}}\right\}\left[\bar{F}_{1}\left(x_{m}\right) \bar{F}_{2}\left(x_{m}\right)\right]^{R_{m}}$

where $x_{i}=x_{i: m: n}$ for simplicity of notation, $\bar{F}_{k}(x)=1-F_{k}(x)$ and $C$ is a constant doesn't depend on the parameters.

\section{Maximum likelihood estimation}

From (1), (2) and (4), the likelihood function ignoring the normalized constant can be written as follows

$L=\left(\alpha_{1} \cdot \beta_{1}\right)^{m_{1}}\left(\alpha_{2} \cdot \beta_{2}\right)^{m_{2}} \prod_{i=1}^{m_{1}} z_{1 i} u_{1 i}^{\beta_{1}-1}\left[1-u_{2 i}{ }^{\beta_{2}}\right] \prod_{i=1}^{m_{2}} z_{2 i} u_{2 i}^{\beta_{2}-1}\left[1-u_{1 i}^{\beta_{1}}\right] \prod_{i=1}^{J}\left[\left(1-u_{1 i}^{\beta_{1}}\right)\left(1-u_{2 i}^{\beta_{2}}\right)\right]^{R_{i}} \times\left[\left(1-u_{1 m}^{\beta_{1}}\right)\left(1-u_{2 m}^{\beta_{2}}\right)\right]^{R_{m}}$

where $u_{k i}=u_{k i}\left(\alpha_{k}\right)=1-e^{-\alpha_{k} \cdot x_{i}}, z_{k i}=z_{k i}\left(\alpha_{k}\right)=e^{-\alpha_{k} \cdot x_{i}}$ and $k=1,2$ and the log-likelihood function is

$$
\begin{aligned}
\ln L= & m_{1}\left(\ln \alpha_{1}+\ln \beta_{1}\right)+m_{2}\left(\ln \alpha_{2} \ln \beta_{2}\right)+\sum_{i=1}^{m_{1}}\left(\left(\beta_{1}-1\right) \ln \left(u_{1 i}\right)+\ln \left[1-u_{2 i}^{\beta_{2}}\right]-\alpha_{1} \cdot x_{i}^{\theta_{1}}\right) \\
& +\sum_{i=1}^{m_{2}}\left(\left(\beta_{2}-1\right) \ln \left(u_{2 i}\right)+\ln \left[1-u_{1 i}^{\beta_{1}}\right]-\alpha_{2} \cdot x_{i}^{\theta_{2}}\right)+\sum_{i=1}^{D} R_{i} \cdot \ln \left[\left(1-u_{1 i}{ }^{\beta_{1}}\right)\left(1-u_{2 i}{ }^{\beta_{2}}\right)\right]+R_{m} \cdot \ln \left[\left(1-u_{1 m}^{\beta_{1}}\right)\left(1-u_{2 m}^{{ }^{\beta_{2}}}\right)\right]
\end{aligned}
$$

The first order derivations of (6) with respect to $\alpha_{k}$ and $\beta_{k}, k=1,2$ are given, respectively, by

$\frac{\partial \ln L}{\partial \alpha_{k}}=\frac{m_{k}}{\alpha_{k}}-\sum_{i=1}^{m_{k}} x_{i}+\left(\beta_{k}-1\right) \sum_{i=1}^{m_{k}} v_{k i}+\sum_{i=1}^{m_{3-k}} s_{k i}+\sum_{i=1}^{J} R_{i} s_{k i}+R_{m} s_{k m}$,

and

$\frac{\partial \ln L}{\partial \beta_{k}}=\frac{m_{k}}{\beta_{k}}+\sum_{i=1}^{m_{k}} \ln \left(u_{k i}\right)+\sum_{i=1}^{m_{3-k}}\left(1-u_{k i}^{-\beta_{k}}\right)^{-1} \cdot \ln \left(u_{k i}\right)+\sum_{i=1}^{J} R_{i} \cdot\left(1-u_{k i}^{-\beta_{k}}\right)^{-1} \cdot \ln \left(u_{k i}\right)+R_{m} \cdot\left(1-u_{k m}^{-\beta_{k}}\right)^{-1} \cdot \ln \left(u_{k m}\right)$.

where

$v_{k i}=v_{k i}\left(\alpha_{k}\right)=\left(x_{i} \cdot z_{k i} / u_{k i}\right), s_{k i}=s_{k i}\left(\alpha_{k}, \beta_{k}\right)=\left(\beta_{k} x_{i} \cdot z_{k i} u_{k i}^{\beta_{k}-1} /\left(u_{k i}^{\beta_{k}}-1\right)\right)$. Equating the first derivations in (7) to zero, one can obtain the MLE of the unknown parameters $\alpha_{1}, \alpha_{2}, \beta_{1}$ and $\beta_{2}$. As it seems, the system of non-linear equations (7) has no closed form solution in $\alpha_{1}, \alpha_{2}, \beta_{1}$ and $\beta_{2}$. So a numerical method technique is required for computing the MLE of the parameters $\alpha_{1}, \alpha_{2}, \beta_{1}$ and $\beta_{2}$.

The asymptotic variance-covariance matrix for $\alpha_{1}, \alpha_{2}, \beta_{1}$ and $\beta_{2}$ can be obtained by inverting the information matrix with the elements that are negative of the expected values of the second order derivatives of logarithms of the likelihood functions. Cohen (1965) concluded that the approximate variance covariance matrix may be obtained by replacing expected values by their MLEs. Now the approximate sample information matrix will be

$\boldsymbol{I}(\hat{\boldsymbol{\theta}})=\left[\begin{array}{cccc}-\frac{\partial^{2} \ln L}{\partial \alpha_{1}^{2}} & 0 & -\frac{\partial^{2} \ln L}{\partial \alpha_{1} \partial \beta_{1}} & 0 \\ 0 & -\frac{\partial^{2} \ln L}{\partial \alpha_{2}^{2}} & 0 & -\frac{\partial^{2} \ln L}{\partial \alpha_{2} \partial \beta_{2}} \\ -\frac{\partial^{2} \ln L}{\partial \beta_{1} \partial \alpha_{1}} & 0 & -\frac{\partial^{2} \ln L}{\partial \beta_{1}^{2}} & 0 \\ 0 & -\frac{\partial^{2} \ln L}{\partial \beta_{2} \partial \alpha_{2}} & 0 & -\frac{\partial^{2} \ln L}{\partial \beta_{2}^{2}}\end{array}\right]_{\hat{\alpha}_{1}, \hat{\alpha}_{2}, \hat{\beta}_{1}, \hat{\beta}_{2}}$

The elements of the $4 \times 4$ matrix, $I_{i j}(\theta), i, j=1,2, . ., 4$ can be obtained as follows

$$
\begin{aligned}
\frac{\partial^{2} \ln L}{\partial \alpha_{k}^{2}}= & -\frac{m_{1}}{\alpha_{k}^{2}}-\left(\beta_{k}-1\right) \sum_{i=1}^{m_{k}} x_{i} v_{k i} u_{k i}^{-1}+\sum_{i=1}^{m_{3-k}} x_{i} s_{k i}\left[\left(\left(\beta_{k}-1\right) z_{k i} u_{k i}^{-1}-1\right)-\beta_{k} u_{k i}^{\beta_{k}-1} z_{k i}\left(u_{k i}^{\beta_{k}}-1\right)^{-1}\right] \\
& +\sum_{i=1}^{J} R_{i} x_{i} s_{k i}\left[\left(\left(\beta_{k}-1\right) z_{k i} u_{k i}^{-1}-1\right)-\beta_{k} u_{k i}^{\beta_{k}-1} z_{k i}\left(u_{k i}^{\beta_{k}}-1\right)^{-1}\right]+R_{m} x_{m} s_{k m}\left[\left(\left(\beta_{k}-1\right) z_{k m} u_{k m}^{-1}-1\right)-\beta_{k} u_{k m}^{\beta_{k}-1} z_{k m}\left(u_{k m}^{\beta_{k}}-1\right)^{-1}\right], \\
\frac{\partial^{2} \ln L}{\partial \beta_{k}^{2}}= & -\frac{m_{k}}{\beta_{k}^{2}}-\sum_{i=1}^{m_{3-k}}\left(1-u_{k i}^{-\beta_{k}}\right)^{-2} u_{k i}^{-\beta_{k}} \ln \left(u_{k i}\right)^{2}-\sum_{i=1}^{J} R_{i}\left(1-u_{k i}^{-\beta_{k}}\right)^{-2} u_{k i}^{-\beta_{k}} \ln \left(u_{k i}\right)^{2}-R_{m}\left(1-u_{k i}^{-\beta_{k}}\right)^{-2} u_{k m}^{-\beta_{k}} \ln \left(u_{k m}\right)^{2},
\end{aligned}
$$

and

$\frac{\partial^{2} \ln L}{\partial \alpha_{k} \partial \beta_{k}}=\sum_{i=1}^{m_{k}} v_{k i}+\sum_{i=1}^{m_{3-k}} \beta_{k}^{-1} s_{k i}\left[1-\beta_{k} \ln \left(u_{k i}\right)\left(u_{k i}^{\beta_{k}}-1\right)^{-1}\right]+\sum_{i=1}^{J} R_{i} \beta_{k}^{-1} s_{k i}\left[1-\beta_{k} \ln \left(u_{k i}\right)\left(u_{k i}^{\beta_{k}}-1\right)^{-1}\right]+R_{m} \beta_{k}^{-1} s_{k m}\left[1-\beta_{k} \ln \left(u_{k m}\right)\left(u_{k m}^{\beta_{k}}-1\right)^{-1}\right]$

where $w_{k i}=w_{k i}\left(\alpha_{k}, \beta_{k}\right)=\left(\alpha_{k} \cdot\left(\beta_{k}-1\right) x_{i} \cdot z_{k i} u_{k i}^{-1}-\alpha_{k} x_{i}+1\right), k=1,2$. Using the independence of the latent failure times 


$$
\begin{aligned}
& \left.X_{1 i}, X_{2 i}, i=1, \ldots, n \text {, we can obtain the relative risk rate due to a particular cause (say, cause } 1\right) \text { as follows } \\
& \pi_{1}=P\left(X_{1 i} \leq X_{2 i}\right)=\int_{0}^{\infty} f_{1}(x) \cdot \bar{F}_{2}(x) d x \\
& \quad=1-\alpha_{1} \cdot \beta_{1} \int_{0}^{\infty} \cdot e^{-\alpha_{1} x} \cdot\left(1-e^{-\alpha_{1} \cdot x}\right)^{\beta_{1}-1} \cdot\left(1-e^{-\alpha_{2} x}\right)^{\beta_{2}} \cdot d x
\end{aligned}
$$

use the binomial expansion of $\left(1-e^{-\alpha_{2} \cdot x}\right)^{\beta_{2}}$, we have

$\pi_{1}==1-\alpha_{1} \cdot \beta_{1} \sum_{i=0}^{\infty}\left(\begin{array}{c}\beta_{2} \\ j\end{array}\right)(-1)^{i} \int_{0}^{\infty} e^{-\alpha_{1} x} \cdot\left(1-e^{-\alpha_{1} x}\right)^{\beta_{1}-1} e^{-i \cdot \alpha_{2} x} \cdot d x$

use the transformation $y=e^{-\alpha_{1} \cdot x}$, we have

$\pi_{1}=1-\beta_{1} \sum_{i=0}^{\infty}\left(\begin{array}{c}\beta_{2} \\ j\end{array}\right)(-1)^{i} B\left(\frac{i \cdot \alpha_{2}}{\alpha_{1}}+1, \beta_{1}\right)$

Once $\pi_{1}$ is computed, we determine $\pi_{2}$ using the relation $\pi_{2}=1-\pi_{1}$. As the integral in the right side of (9) has no analytical solution, we have to use a numerical technique to solve the integral. According to the invariance property of the MLE, the MLE of the relative risk rates $\pi_{1}$, can be obtained by replacing the MLE of $\alpha_{1}, \alpha_{2}, \beta_{1}$ and $\beta_{2}$ in (9). Based on the above results, When $\beta_{1}=\beta_{2}=1$, the MLE's of $\alpha_{1}$ and $\alpha_{2}$ and the relative risk rates $\pi_{1}$ and $\pi_{2}$, corresponds to the results of the exponential distribution obtained by Hemmati and Khorram [11], when the cause of failure is known.

\section{Asymptotic confidence intervals}

In this section we derive the confidence intervals of the vector of the unknown parameters $\boldsymbol{\theta}=\left(\alpha_{1}, \alpha_{2}, \beta_{1}, \beta_{2}\right)$. Based on the asymptotic distribution of the MLE of the parameters, it is known that

$(\hat{\boldsymbol{\theta}}-\boldsymbol{\theta}) \longrightarrow N_{4}\left(0, I^{-1}(\boldsymbol{\theta})\right)$

where $I(\theta)$ is the Fisher information matrix. The elements of $4 \times 4$ matrix $I_{i j}(\theta)$ can be approximated by $I_{i j}(\hat{\theta})$, where

$I_{i j}(\hat{\theta})=-\left.\frac{\partial^{2} \ln L}{\partial \theta_{i} \partial \theta_{j}}\right|_{\theta=\hat{\theta}}, i, j=1,2, . ., 4$

and $\partial^{2} \ln L / \partial \theta_{i} \partial \theta_{j}$ is the second derivations obtained in (8). The 100(1- $\left.\alpha\right)$ approximate confidence intervals of the vector of the unknown parameters $\boldsymbol{\theta}=\left(\alpha_{1}, \alpha_{2}, \beta_{1}, \beta_{2}\right)$ can be obtained as follows

$\hat{\theta}_{j} \pm z_{\alpha / 2} \cdot \sqrt{\operatorname{var}\left(\hat{\theta}_{j}\right)}, j=1, \ldots, 4$.

where $\operatorname{var}\left(\hat{\theta}_{j}\right)$ is the elements on the main diagonal of $I^{-1}(\hat{\boldsymbol{\theta}})$ and $z_{\alpha / 2}$ is the upper $(\alpha / 2)$-th percentile of a standard normal distribution.

\section{Goodness of fit}

We now discuss the problem of testing goodness of fit of a competing risks model when the causes of failures follow the GED against the ED to illustrate whether the GED can better fit a real data set rather than the ED studied by Hemmati and Khorram [11]. Because the ED can be derived as a special case of the GED, the likelihood ratio test will be used to test the adequacy of generalized exponential distributions competing risks. The null and alternative hypotheses are

$H_{0}: \beta_{1}=\beta_{2}=1$, the causes of death follow ED,

$H_{0}: \beta_{1} \neq \beta_{2} \neq 1$, the causes of death follow GED.

The test statistic is the ratio of the likelihood of $H_{0}$ and the likelihood of $H_{1}$, and given by the expression

$\Lambda=L\left(\boldsymbol{\theta}_{0}\right) / L(\boldsymbol{\theta})$

where $\theta$ is a vector of parameters, and $\theta_{0}$ is a subset of $\theta$. Under the null hypothesis the log-likelihood ratio test statistic is

$X_{L}=-2 \ln (\Lambda)=2\left(\mathcal{L}(\boldsymbol{\theta})-\mathcal{L}\left(\boldsymbol{\theta}_{0}\right)\right)$

where $\mathcal{L}(\boldsymbol{\theta})$ and $\mathcal{L}\left(\boldsymbol{\theta}_{0}\right)$ are the log-likelihood functions under $H_{1}$ and $H_{0}$, respectively. Asymptotically, the test statistic is distributed as a chi-squared distribution with $v$ degree of freedom. Now, we can write the log-likelihood ratio test statistics as follows

$X_{L}=2\left(\mathcal{L}_{G E D}-\mathcal{L}_{E D}\right)$ 
where $\mathcal{L}_{E D}$ and $\mathcal{L}_{G E D}$ are the log-likelihood functions under $H_{0}$ and $H_{1}$, respectively, after replacing the unknown parameters with their MLE.

For comparison purposes between the candidate models, we can use two model criterion selection, the Akaike information criterion (AIC) (Akaike [13]) and Bayes information criterion (BIC) (Schwarz [12]) defined as

$\mathrm{AIC}=-2 \mathcal{L}+2 p$ and $\mathrm{BIC}=-2 \mathcal{L}+p \cdot \ln (n)$

where $p$ is the number of parameters in the model, and $\mathcal{L}$ is the maximized value of the likelihood function for the model. As a model selection criterion, the researcher should choose the model that minimizes AIC and BIC.

\section{Numerical results}

In this section, we analyze one data set which was originally analyzed by Hoel [6] and later by Kundu et al [7], Pareek et al [4], Cramer and Schmiedt [9], Hemmati and Khorram [11] and Ashour and Nassar [17]. The data was obtained from a laboratory experiment in which male mice received a radiation dose of 300 roentgens at 35 days to 42 days (5-6 weeks) of age. The cause of death for each mouse was determined by reticulum cell sarcoma as cause 1 and other causes of death as cause 2, there were $n=77$ observations remain in the analysis. Using the censoring scheme $m=25$ and $R_{1}=R_{2}=\ldots=R_{25}=2$, the progressive type-II censored sample from the original data is given by

$(40,2),(42,2),(62,2),(163,2),(179,2),(206,2),(222,2),(228,2),(252,2),(259,2),(318,1),(385,2),(407,2),(420$, $2),(462,2),(517,2),(517,2),(524,2),(525,1),(536,1),(558,1),(605,1),(612,1),(620,2),(621,1)$. All of the computations were performed using MATHCAD program version 2007.

The first component denotes the life time and the second component indicate the cause of failure.

Example 1: Considering $T=550$, then $J=20, m_{1}=7$ and $m_{2}=18$. From the above data, the MLEs of the unknown parameters, the corresponding approximate $95 \%$ two sided confidence intervals distributions, the log-likelihood values $(\mathcal{L})$, AIC and BIC shown given in table (1).

Table 1: The MLE and Approximate 95\% Two Sided Confidence Intervals of the Parameters in Each Model (ED and GED).

\begin{tabular}{|c|c|c|c|c|c|c|c|}
\hline \multirow{2}{*}{ Model } & \multicolumn{4}{|c|}{ Estimates } & \multicolumn{3}{|c|}{ Statistics } \\
\hline & $\alpha_{1}$ & $\alpha_{2}$ & $\beta_{1}$ & $\beta_{2}$ & $\mathcal{L}$ & $\mathrm{AIC}$ & $\mathrm{BIC}$ \\
\hline ED & $\begin{array}{c}0.000239 \\
(0.00006,0.00042)\end{array}$ & $\begin{array}{c}0.000615 \\
(0.00033,0.0009)\end{array}$ & -- & -- & -215.9 & 435.9 & 440.6 \\
\hline GED & $\begin{array}{c}0.0047 \\
(0.0021,0.007)\end{array}$ & $\begin{array}{c}0.0011 \\
(0.0003,0.002)\end{array}$ & $\begin{array}{c}25.885 \\
(0,62.47)\end{array}$ & $\begin{array}{c}1.527 \\
(0.728,2.326)\end{array}$ & -204.4 & 416.8 & 426.3 \\
\hline
\end{tabular}

and the relative risk due to cause one for ED and GED are 0.28 and 0.5577 , respectively. The value of the likelihood ratio test statistic is $X_{L}=24.844$, and the corresponding p-value is 0.00005 .

Example 2: Now we use the same data, but use $T=610$ instead of $T=550$, while $\mathrm{m}$ and $R_{i}$ 's are same as before, then $J=22, m_{1}=7$ and $m_{2}=18$. the MLEs of the unknown parameters, the corresponding approximate $95 \%$ two sided confidence intervals distributions, the log-likelihood values, AIC and BIC shown given in table (2).

Table 2: The MLE and Approximate 95\% Two Sided Confidence Intervals of the Parameters in Each Model (ED and GED).

\begin{tabular}{|c|c|c|c|c|c|c|c|}
\hline \multirow{2}{*}{ Model } & \multicolumn{4}{|c|}{ Estimates } & \multicolumn{3}{|c|}{ Statistics } \\
\hline & $\alpha_{1}$ & $\alpha_{2}$ & $\beta_{1}$ & $\beta_{2}$ & $\mathcal{L}$ & AIC & BIC \\
\hline ED & $\begin{array}{c}0.000241 \\
(0.00006,0.000419)\end{array}$ & $\begin{array}{c}0.000619 \\
(0.00033,0.000904)\end{array}$ & -- & -- & -215.8 & 435.7 & 440.4 \\
\hline GED & $\begin{array}{c}0.0049 \\
(0.0022,0.008)\end{array}$ & $\begin{array}{c}0.0011 \\
(0.0003,0.002)\end{array}$ & $\begin{array}{c}28.09 \\
(0,68.109)\end{array}$ & $\begin{array}{c}1.5433 \\
(0.735,2.352)\end{array}$ & -203.8 & 415.8 & 425.1 \\
\hline
\end{tabular}

and the relative risk due to cause one for ED and GED are 0.28 and 0.5597 , respectively. The value of the likelihood ratio test statistic is $X_{L}=25.63$, and the p-value is 0.00003 .

The analysis of the previous real data set demonstrates the importance and usefulness of adaptive type-II progressive hybrid censoring scheme and inferential procedures based on them. From example 1 and 2 , it is observed that $T$ plays a major role in the estimation and for the construction of the corresponding confidence intervals, because when $T$ increases some additional information is gathered. We also conclude that based on the values of $X_{L}$, the p-value, AIC and BIC, the GED fits the data better than ED. We have observed that the assumptions that the generalized exponential distributions may be used to analyze this set of real data better that the exponential distribution. 


\section{References}

[1] A. C. Cohen, Maximum likelihood estimation in the Weibull distribution based on complete and censored samples, Technometrics 5 (1965) 579-588. http://dx.doi.org/10.1080/00401706.1965.10490300.

[2] A.Childs, B. Chandrasekar, N. Balakrishnan, Exact likelihood inference for an exponential parameter under progressive hybrid censoring. In: Statistical Models and Methods for Biomedical and Technical Systems, F. Vonta, M. Nikulin, N. Limnios, and C. Huber-Carol, (Eds). Birkhäuser, Boston, (2008) 323-334. http://dx.doi.org/10.1007/978-0-8176-4619-6_23.

[3] B. Epstein, Truncated life-test in exponential case. Annals of Mathematical Statistic, 25 (1954) 555 - 564. http://dx.doi.org/10.1214/aoms/1177728723.

[4] B. Pareek, D. Kundu, S. Kumar, On progressively censored competing risks data for Weibull distribution, Computational Statistics and Data Analysis, 53(2009) 4083-4094. http://dx.doi.org/10.1016/j.csda.2009.04.010.

[5] C. T. Lin, H. K. T. Ng, P. S. Chan, Statistical inference of type-II progressively hybrid censored data with Weibull lifetimes, Communications in Statistics-Theory and Methods, 38 (2009) 1710-1729. http://dx.doi.org/10.1080/03610920902850069.

[6] D. G. Hoel, A representation of mortality data by competing risks, Biometrics, 28 (1972) 475-488. http://dx.doi.org/10.2307/2556161.

[7] D. Kundu, N. Kannan, N. Balakrishnan, Analysis of progressively censored competing risks data, Handbook of Statistics, 23 (2004), eds., Balakrishnan, N. and Rao, C.R., Elsevier, New York.

[8] D. Kundu, A. Joarder, Analysis of type-II progressively hybrid censored data. Computational Statistics \& Data Analysis 50, (2006) 25092528. http://dx.doi.org/10.1016/j.csda.2005.05.002.

[9] E. Cramer, A.B. Schmiedt, Progressively type-II censored competing risks data from Lomax distribution, Computational Statistics and Data Analysis 55 (2011) 1285-1303. http://dx.doi.org/10.1016/j.csda.2010.09.017.

[10] F. Hemmati, E. Khorram, Statistical Analysis of the Log-Normal Distribution under Type-II Progressive Hybrid Censoring Schemes. Communications in Statistics-Theory and Methods, 42 (2013) 52-75.

[11] F. Hemmati, E. Khorram, Bayesian analysis of the adaptive type-II progressively hybrid censoring scheme in presence of competing risks, Proc. ICCS-11, Lahore, Pakistan, 21(2011) 181-194.

[12] G. Schwarz, Estimating the dimension of a model, The Annals of Statistics, 6(2) (1978) 461-4. http://dx.doi.org/10.1214/aos/1176344136.

[13] H. Akaike, Fitting autoregressive models for regression, Annals of Institute of Statistical Mathematics, 21 (1969) $243-7$. http://dx.doi.org/10.1007/BF02532251.

[14] H.K.T. Ng, D. Kundu, P.S. Chan, Statistical analysis of exponential lifetimes under an adaptive Type-II progressively censoring scheme, Naval Research Logistics, 56 (2009) 687-698. http://dx.doi.org/10.1002/nav.20371.

[15] M. A. W. Mahmoud, A. A. Soliman, A. H. Abd Ellah, R.M. El-Sagheer, Estimation of generalized Pareto under an adaptive type-II progressive censoring, Intelligent Information Management, 5 (2013) 73-83. http://dx.doi.org/10.4236/iim.2013.53008.

[16] R. D. Gupta, D. Kundu, Generalized Exponential Distributions, Australian and New Zealand Journal of Statistics, $41(2)$ (1999) $173-188$. http://dx.doi.org/10.1111/1467-842X.00072.

[17] S.K. Ashour, M.M.A. Nassar, Analysis of exponential distribution under adaptive type-I progressive hybrid censored competing risks data, Pakistan Journal of Statistics and Operation Research, 10 (2) (2014) 229-245. 